\title{
Liquid crystal pretilt control by inhomogeneous surfaces
}

\author{
Jones T. K. Wan, ${ }^{1, *}$ Ophelia K. C. Tsui, ${ }^{1, \dagger}$ Hoi-Sing Kwok, ${ }^{2}$ and Ping Sheng ${ }^{1}$ \\ ${ }^{1}$ Department of Physics and Institute of Nano Science and Technology, Hong Kong University of Science and Technology, Clear Water \\ Bay, Kowloon, Hong Kong \\ ${ }^{2}$ Department of Electrical and Electronic Engineering, Hong Kong University of Science and Technology, Clear Water Bay, Kowloon, \\ Hong Kong
}

(Received 23 May 2005; published 30 August 2005)

\begin{abstract}
We consider the pretilt alignment of a nematic liquid crystal (LC) on inhomogeneous surface patterns comprising patches of homeotropic alignment domains in a matrix favoring homogeneous alignment, or vice versa. We found that the resultant LC pretilt generally increases continuously from the homogeneous limit to the homeotropic limit as the area fraction of the homeotropic region increases from 0 to 1 . For any given homeotropic area fraction, the variations are qualitatively different depending on how the distance between adjacent patches compares to the extrapolation lengths of the anchoring domains. Our results agree with those previously found in stripe patterns. The present finding provides useful guidelines for designing inhomogeneous alignment surfaces for variable LC pretilt control.
\end{abstract}

DOI: 10.1103/PhysRevE.72.021711

PACS number(s): 61.30.Hn, 61.30.Eb, 61.30.Dk

The study of inhomogeneous surfaces for liquid crystal (LC) alignment has witnessed a rapid growth in recent years [1-10]. Experiments showed that these surfaces could have unique applications as in the making of multistable display devices [7] and the control of LC pretilt alignment $[5,10]$. These types of alignment surfaces generally comprise two kinds of domains favoring different LC orientations. The majority of theoretical treatments dealt with domains arranged in stripes [1-4,6] or checkerboards [7-9]. No systematic calculation has been carried out for a patchy pattern to our knowledge, which is more likely achieved by nonlithographic techniques that would be less costly [5]. In this paper, we report results from an extensive simulation study of the alignment of nematic LC on surfaces containing patches of homogeneous or homeotropic alignment domains, with one embedded in the matrix of the other.

The alignment patterns considered are periodic with unit cells like those shown in Fig. 1 with different area fractions $p$ of the homeotropic domains. We consider a cubic simulation cell with sides of unity filled with a nematic LC to which periodic boundary conditions are imposed. The lower $(z=0)$ surface is the patterned surface as shown in Fig. 1, and the upper surface is free (i.e., the surface anchoring energy is zero there). The use of a free upper surface is to emulate the simulation cell as one with infinite thickness. The choice of unity for the thickness of the simulation cell is justified $a$ posteriori from the fact that the solution to the equilibrium LC configuration is always uniform at the upper boundary. If each type of domain has surface energy $W_{i}(\theta, \phi)(i=1,2)$, the unit-area surface energy $F_{s}$ would be

\footnotetext{
*Electronic mail: jwan@ust.hk

†Electronic mail: phtsui@ust.hk
}

$$
F_{s}(\theta(\boldsymbol{r}), \phi(\boldsymbol{r}))=\delta(z) \sum_{i=1}^{2} f_{i}(x, y) W_{i}(\theta(\boldsymbol{r}), \phi(\boldsymbol{r}))
$$

Here, $\boldsymbol{r}=(x, y, z)$ is a position vector in the simulation cell; the LC director field is denoted by $\boldsymbol{n}(\boldsymbol{r})$ $\equiv(\cos \theta \cos \phi, \cos \theta \sin \phi, \sin \theta)$, where $\theta(\boldsymbol{r})$ and $\phi(\boldsymbol{r})$ are, respectively, the pretilt angles (measured from the surface) and azimuthal angles of the LC director; $f_{i}(x, y)$ is one if $(x, y)$ belongs to domain $i$ and zero otherwise so that the area fraction of domain $i$ is obtainable by integrating $f_{i}(x, y)$ over the $z=0$ unit surface. Since our focus is on the LC pretilt alignment produced by the inhomogeneous surface, we impose anisotropy in the $x-y$ plane, i.e., both domains prefer the same azimuthal orientation along, say, $\phi_{0}$. We further assume the local preferred pretilt alignment of domain $1, \theta_{01}=88^{\circ}$ and that of domain $2, \theta_{02}=6^{\circ}$ to lift the foreseeable degeneracy between the $\phi(\boldsymbol{r})=\phi_{0}$ and $\phi(\boldsymbol{r})=\phi_{0}+180^{\circ}$ states. With these assumptions, the azimuthal orientation of the LC is constant in space and equal to $\phi_{0}$. Hence the surface energy depends only on $\theta(\boldsymbol{r})$. We use the Rapini-Papoular form of

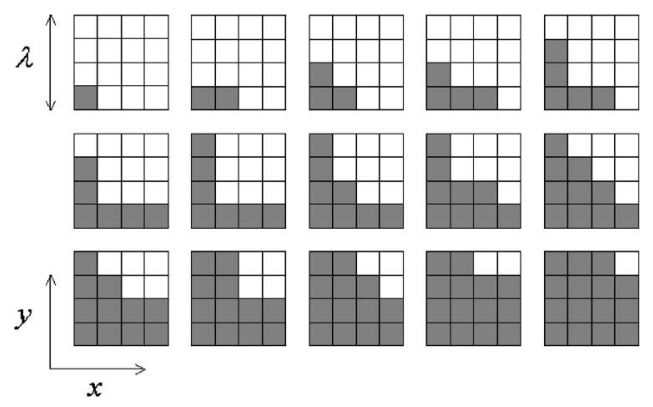

FIG. 1. Patterns used to simulate the inhomogeneous surface. Filled squares stand for the homeotropic domains; unfilled squares stand for the homogeneous domains. Periodic boundary conditions are imposed in the $x$ and $y$ directions. 
(a)
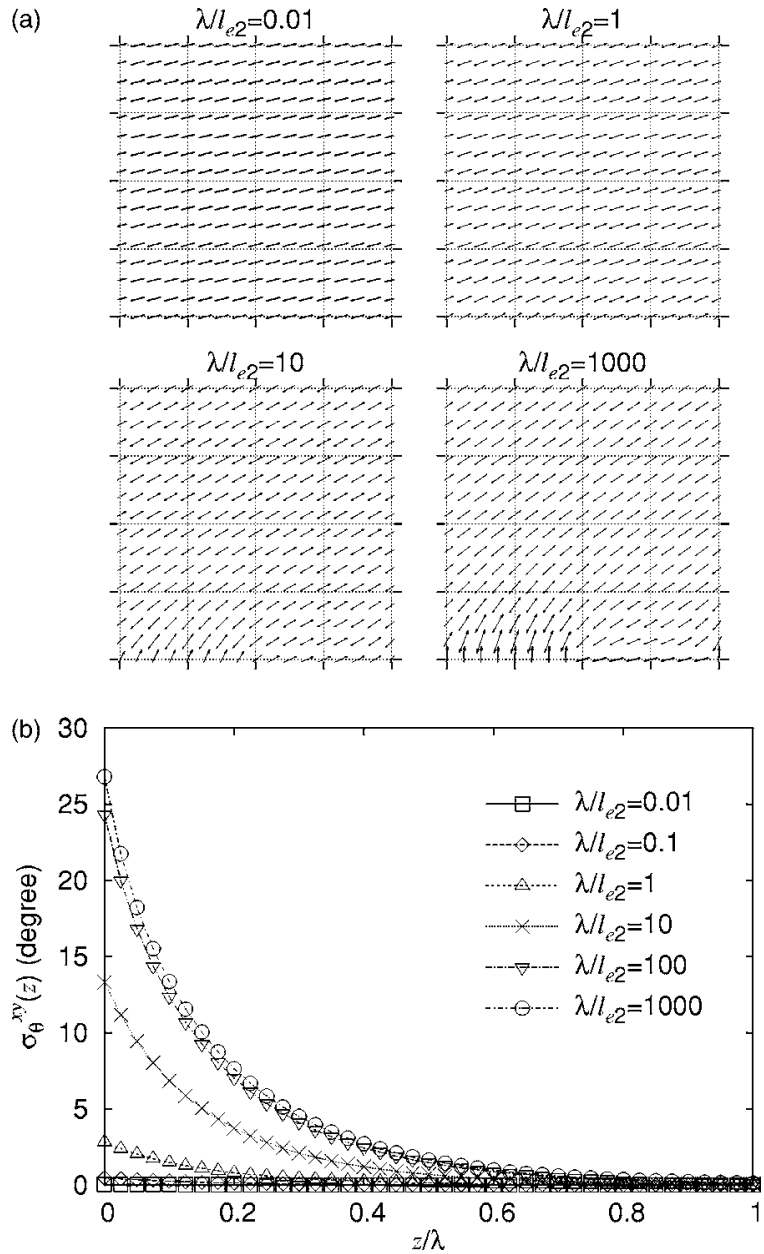

FIG. 2. (a) Liquid crystal director field in the $y=0$ plane for $\lambda / \ell_{e 2}=0.01,1,10$, and 1000. (b) Profiles of the standard deviation of the LC pretilt along $z$ for the same $\lambda / \ell_{e 2}$ 's shown in (a). It was assumed here that $p=0.125$ and $W_{\theta 1}=W_{\theta 2}$ or $\ell_{e 1}=\ell_{e 2}$.

the LC surface energy [8], $W_{i}\left(\theta, \phi_{0}\right)=\frac{1}{2} W_{\theta i} \sin ^{2}\left(\theta-\theta_{0 i}\right)$ where $W_{\theta i}$ is the polar anchoring strength of domain $i$. For the LC elastic energy density $F_{e}$, we assume the Frank-Oseen form:

$F_{e}(\boldsymbol{n})=\frac{1}{2}\left[K_{11}(\boldsymbol{\nabla} \cdot \boldsymbol{n})^{2}+K_{22}(\boldsymbol{n} \cdot \boldsymbol{\nabla} \times \boldsymbol{n})^{2}+K_{33}(\boldsymbol{n} \times \boldsymbol{\nabla} \times \boldsymbol{n})^{2}\right]$.

Here $K_{11}, K_{22}$, and $K_{33}$ are the splay, twist, and bend elastic constants, respectively, for which the values of the nematic 5CB (4, $n$-pently-4-cyanobiphenyl) are used in simulations [8]: $K_{11}=7.0 \times 10^{-12} \mathrm{~N}, K_{22}=3.5 \times 10^{-12} \mathrm{~N}, K_{33}=1.0$ $\times 10^{-11} \mathrm{~N}$. If the size of the cubic cell is scaled to $\lambda^{3}$ where $\lambda$ denotes the period of the actual surface pattern, the total energy per unit area is given by

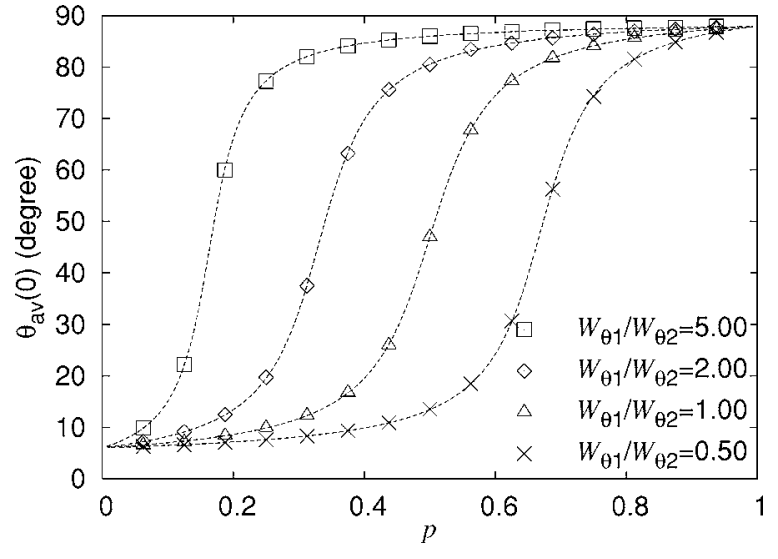

FIG. 3. Simulation results of average pretilt angle $\theta_{\mathrm{av}}(z=0)$ versus $p$ for $\lambda / \ell_{e 2}=0.01$ and various $W_{\theta 1} / W_{\theta 2}=0.5$ (crosses), 1.0 (triangles), 2.0 (diamonds), and 5.0 (squares) are shown. Dashed lines are the simulated $\theta_{\min }$ versus $p$ using Eq. (4) for the same set of $W_{\theta 1} / W_{\theta 2}$.

$$
\begin{aligned}
\frac{F_{\mathrm{tot}}(\boldsymbol{n})}{\lambda^{2}}= & \frac{K_{11}}{\lambda}\left(\int_{0}^{1} \int_{0}^{1} \int_{0}^{1} \widetilde{F}_{e}(\boldsymbol{n}) d x d y d z\right. \\
& \left.+\sum_{i=1}^{2} \int_{0}^{1} \int_{0}^{1} \frac{\lambda}{\ell_{e i}} f_{i}(x, y) \frac{\sin ^{2}\left(\theta-\theta_{0 i}\right)}{2} d x d y\right),
\end{aligned}
$$

where $\widetilde{F}_{e} \equiv F_{e} / K_{11}$, and $\ell_{e i} \equiv K_{11} / W_{\theta i}$ is the extrapolation length of domain $i$. Equation (3) shows that for the same LC material, the energy density of the system generally varies with $\lambda$ according to $1 / \lambda$. However, the critical parameter determining the LC configuration should be the ratio $\lambda p_{i} / \ell_{e i}$ (where $p_{i}=p$ and $1-p$ for $i=1$ and 2, respectively): if either $\lambda p_{i} / \ell_{e i}$ is $\ll 1$, the elastic energy would dominate the surface energy, and vice versa in the opposite limit. For typical alignment surfaces, the polar anchoring energy is $10^{-4}-10^{-3} \mathrm{~J} \mathrm{~m}^{-2}$ and hence $\ell_{e i} \approx 0.01-0.1 \mu \mathrm{m}$. We determine the equilibrium LC director field by minimizing Eq. (3) with respect to $\boldsymbol{n}(\boldsymbol{r})$ numerically as in Ref. [9].

Shown in Fig. 2(a) are the LC director field in the $y=0$ plane for $\lambda / \ell_{e 1}=\lambda / \ell_{e 2}=0.01,1,10$, and 1000. In obtaining these data, it was assumed that $p=0.125$. But in fact the results are qualitatively independent of the precise values of $p$ and $W_{\theta 1} / W_{\theta 2}$. As seen, the LC alignment is uniform for $\lambda / \ell_{e 2} \ll 1$ (note that $p_{2}=0.875$ is $\approx 1$ ) but becomes increasingly inhomogeneous with increasing $\lambda / \ell_{e 2}$ when $\lambda / \ell_{e 2}$ is $\geqslant 1$. In the latter cases, the inhomogeneity is the biggest at the patterned surface (i.e., $z=0$ ) and relaxes to uniformity within a distance much less than $\lambda / 4$. We quantify this observation by plotting $\sigma_{\theta}^{x y}(z)$, the standard deviation of $\theta(\boldsymbol{r})$ over the $x-y$ plane at $z$, against $z$ for the various values of $\lambda / \ell_{e 2}$ examined in Fig. 2(b). As seen, when $\lambda / \ell_{e 2} \ll 1$ $(=0.01,0.1)$, variation of the LC pretilt in the $x-y$ plane is negligibly small for all $z$, consistent with the data shown in Fig. 2(a). But as $\lambda / \ell_{e 2}$ increases toward $1, \sigma_{\theta}^{x y}(0)$ increases notably, and steadily approaches $\sim 27^{\circ}$ as $\lambda / \ell_{e 2}$ continues to increase toward 1000. We postpone discussion of the noted asymptotic value of $\sigma_{\theta}^{x y}(0)$ until later. Despite the large varia- 
tions in $\sigma_{\theta}^{x y}(0)$, all curves display similar decays toward 0 within $z=\lambda$. Least-square fits to the data reveal that the data of Fig. 2 can be described very well by an exponential function with decay length $\sim \lambda / 2 \pi$, which agrees with the above observation and previous results found in stripe surface patterns possessing zenithal inhomogeneity [3].

We first discuss the average LC pretilt alignment in the $\lambda p_{i} / \ell_{e i} \ll 1$ limit. Shown in Fig. 3 are the average LC pretilt at $z=0, \theta_{\mathrm{av}}(z=0)$, plotted versus $p$ for fixed $\lambda / \ell_{e 2}=0.01$ and $W_{\theta 1} / W_{\theta 2}$ varied from 0.5 to 5 . As seen, all the curves in Fig. 3 bear the same shape with $\theta_{\mathrm{av}}(0)$ demonstrating a gentle rise from $6^{\circ}$ as $p$ increases from 0 , followed by an abrupt rise to $>70^{\circ}$ whereupon the rise with $p$ becomes gradual and asymptotically approaches a plateau with $\theta_{\mathrm{av}}(0)=88^{\circ}$. For $W_{\theta 1} / W_{\theta 2}=1$, the transition occurs at $p=0.5$, but shifts to smaller (larger) $p$ when $W_{\theta 1} / W_{\theta 2}$ increases (decreases). By adopting a uniform LC director field as found above for this limit, the system must have its energy come solely from the surface energy. Thereby the pretilt angle should just be $\theta_{\min }$, the pretilt angle that minimizes the surface energy term of Eq. (3). It is straightforward to show that $\theta_{\min }$ satisfies

$$
\frac{1}{p}=1-\frac{W_{\theta 1} \sin 2\left(\theta_{\min }-\theta_{01}\right)}{W_{\theta 2} \sin 2\left(\theta_{\min }-\theta_{02}\right)} .
$$

In Fig. 3 are plotted $\theta_{\min }$ deduced from Eq. (4) versus $p$ for the same set of $W_{\theta 1} / W_{\theta 2}$ considered (dashed lines). As seen, the data of $\theta_{\min }$ demonstrates excellent agreement with those of $\theta_{\text {av }}(0)$ obtained from simulations, verifying our interpretation that the LC pretilt should just be $\theta_{\min }$ in this limit.

Next we consider the opposite limit where $\lambda p_{i} / \ell_{e i} \gg 1$. In this case, the director field on the surface is no longer uniform. As Fig. 2 shows, the surface-induced inhomogeneity decays within a distance of $\sim \lambda / 2 \pi$. Hence the homogenized LC alignment in the bulk can be obtained from that at $z=\lambda$. Shown in Fig. 4 are plots of $\theta_{\mathrm{av}}(\lambda)$ versus $p$ for $\lambda / \ell_{e 2}$ $=1000$ and the same set of $W_{\theta 1} / W_{\theta 2}$ investigated in Fig. 3. In the same figure are shown the corresponding data at $z=0$ (solid line) for comparison. As seen, the average LC pretilts at $z=0$ and $z=\lambda$ are independent of $W_{\theta 1} / W_{\theta 2}$. It is interesting to note that the $z=0$ data demonstrate a simple linear rise with $p$ from $6^{\circ}$ to $88^{\circ}$. This is because when $\lambda / \ell_{e} \gg 1$, the surface energy dominates the elastic energy in the LC system. One may thus assume the LC director to be exactly following that of the local preferred direction of the alignment surface whereby $\theta_{\text {av }}(0)$ should be given by the algebraic average of the local preferred orientation over the surface, i.e., $p \theta_{01}+(1-p) \theta_{02}$. For $p=0.125$, namely, the case exemplified in Fig. $2, \theta_{\mathrm{av}}(0)=16.25^{\circ}$ and correspondingly the standard deviation of $\theta(x, y)$ at $z=0$ would be $27.1^{\circ}$, which is in excellent agreement with the value noted previously from Fig. 2 for $\lambda / \ell_{2}=1000$. The slightly larger value of $\theta_{\mathrm{av}}$ in the bulk than that at $z=0$ is attributable to the assumed $K_{33}$ being larger than $K_{11}$, which favors less bending in the expense of more splay deformations in the LC director field [2].

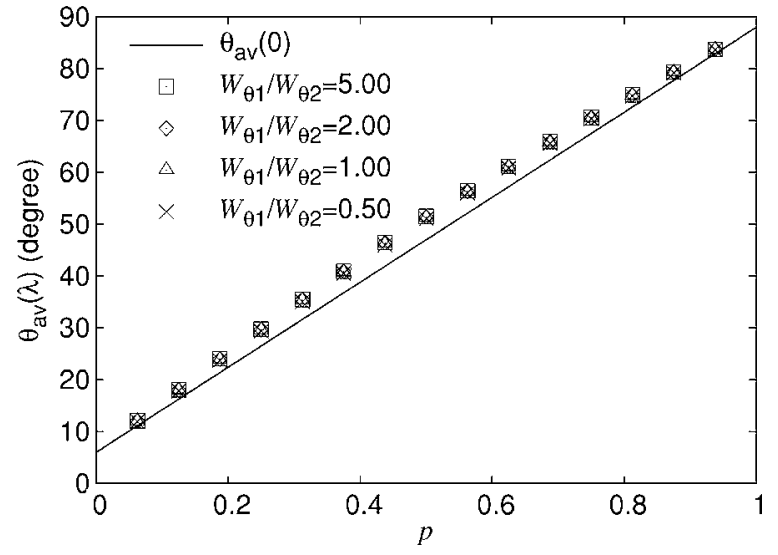

FIG. 4. The average LC pretilt at $z=\lambda$, which is essentially the bulk value, versus $p$ for $\left(\lambda / \ell_{e 2}=1000\right)$ for four different $W_{\theta 1} / W_{\theta 2}$ as indicated. The data for average pretilt at $z=0$ are also drawn (solid line).

In summary, we have considered in detail the pretilt alignment of nematic LC on a periodic patchy surface pattern comprising domains favoring pretilt orientations either along $88^{\circ}$ or $6^{\circ}$. We found that if the pattern period is much smaller than the extrapolation lengths of the two kinds of domains, the LC configuration is uniform with the LC pretilt equal to that minimizing the surface anchoring energy. But if the pattern period is much bigger than the two extrapolation lengths, the LC alignment at the interface copies the inhomogeneity of the surface pattern, which, however, dies out within one pattern period from the surface. The resulting homogenized LC pretilt orientation in the bulk is approximately equal to the average of the local preferred directions of the domains weighted by their area fractions in the pattern. Previously Ong et al. [5] created random inhomogeneous surfaces by depositing a discontinuous metal film on a silane (homeotropic agent) coated corrugated $\mathrm{SiO}_{2}$ (homogeneous) substrate and then subjecting the sample to a glow discharge to remove the silane unprotected by the metal islands, before dissolving the metal in the end. The average LC pretilt on these surfaces was found to increase monotonically from $0^{\circ}$ to $90^{\circ}$ as the average metal film thickness was increased, demonstrating good qualitative agreement with our results. Our results also display good consistency with those of Barbero et al. [3] who obtained analytic solutions for LC alignment on stripe patterns favoring two alternating, dissimilar pretilt orientations (though the two preferred orientations must be sufficiently similar to validate their calculation). As most discussions presented here are general to LC alignment on inhomogeneous surfaces, it is probable that our results would be applicable to more general inhomogeneous patterns with irregular shapes.

We acknowledge support of the Research Grant Council of Hong Kong through Project No. HKUST6115/03E. 
[1] E. Guyon, P. Pieranski, and M. Boix, Lett. Appl. Eng. Sci. 1, 19 (1973).

[2] D. W. Berreman, J. Phys. Colloq. 40, 58 (1979).

[3] G. Barbero, T. Beica, A. L. Alex-Ionescu, and R. Moldovan, J. Phys. II 2, 2011 (1992).

[4] T. Z. Qian and P. Sheng, Phys. Rev. Lett. 77, 4564 (1996).

[5] H. L. Ong, A. J. Hurd, and R. B. Mayer, J. Appl. Phys. 57, 186 (1985).

[6] B. Lee and N. A. Clark, Science 291, 2576 (2001).
[7] J. Kim, M. Yoneya, and H. Yokoyama, Nature (London) 420, 159 (2002).

[8] B. Zhang, F. K. Lee, O. K. C. Tsui, and P. Sheng, Phys. Rev. Lett. 91, 215501 (2003).

[9] O. K. C. Tsui, F. K. Lee, B. Zhang, and P. Sheng, Phys. Rev. E 69, 021704 (2004).

[10] F. K. Lee, B. Zhang, P. Sheng, H. S. Kwok, and O. K. C. Tsui, Appl. Phys. Lett. 85, 5556 (2004). 